Publ. RIMS, Kyoto Univ. Ser. A

Vol. 3 (1967), pp. 85-99

\title{
Control problems of contingent equation
}

By

\section{Norio KIKUCHI*}

\section{Introduction}

In this paper we shall prove the existences of solutions for the Carathéodory type contingent equation and, using this existence theorem, we shall consider a control problem for the contingent equation.

Furthermore we shall extend the existence theorem of optimal control that was considered in [3] to the case of the contingent equation.

The author wishes here to express his thanks to Professor Masuo Hukuhara, who read the original manuscripts and suggested a number of improvements.

\section{Notations and definitions}

The notations used in this paper are the followings.

Let $X$ be a metric space. The distance between two points $x, y$ $\varepsilon X$ is denoted by $\operatorname{dist}(x, y)$. The distance between a point $x \in X$ and a set $A \subset X$ is defined by $\operatorname{dist}(x, A)=\inf \{\operatorname{dist}(x, y) ; y \in A\}$. For $\delta>0$, the $\delta$-neighborhood of a set $A \subset X$ is denoted by

$$
U(A, \delta)=\{x \in X ; \operatorname{dist}(x, A)<\delta\} .
$$

For two compact sets $A, B \subset X$, the distance between $A$ and $B$ is denoted by $\operatorname{Dist}(A, B)$, where $\operatorname{Dist}(A, B)=\inf \{\delta>0 ; U(A, \delta) \supset B$, $U(B, \delta) \supset A\}$. This (Hausdorff) distance makes the set of compact sets into a metric space.

Definition 1. A compact-set (in $X$ ) valued function $F(t)$ defined on a topological space $T$, is said to be upper (resp. lower) semi-

Received March 27, 1967.

Communicated by M. Hukuhara.

* Department of Mathematics, Kôbe University. 
continuous at $t_{0}\left(t_{0} \in T\right)$, if for every $\varepsilon>0$ we can find some neighborhood of $t_{0}$, say $V$, such that $U\left(F\left(t_{0}\right), \varepsilon\right) \supset F(t)($ resp. $U(F(t), \varepsilon)$ $\left.\supset F\left(t_{0}\right)\right)$ for all $t \in V$. When $F(t)$ is upper (resp. lower) semi-continuous at every point of $T, F(t)$ is said to be upper (resp. lower) semi-continuous on T. A function $F(t)$ is said to be continuous at $t_{0}$ (resp. on $T$ ) when $F(t)$ is upper and lower semi-continuous at $t_{0}$ (resp. on $T$ ).

Definition 2. If a compact-set (in $X$ ) valued function $F(t)$ defined on a measurable space $E$ is such that, for every compact $C$ of $X$, the set $\{t \in E ; F(t) \subset C\}$ is measurable, then $F$ is said to be measurable on $E$.

Definition 3. For a sequence of subsets (in $X)\left\{A_{n}\right\} \quad(n=1,2$, ..) we define

and

$$
\begin{aligned}
& \lim _{n \rightarrow \infty} \inf A_{n}=\left\{x \in X ; \lim _{n \rightarrow \infty} \operatorname{dist}\left(x, A_{n}\right)=0\right\} \\
& \lim _{n \rightarrow \infty} \sup A_{n}=\left\{x \in X ; \lim _{n \rightarrow \infty} \operatorname{dist}\left(x, A_{n}\right)=0\right\}
\end{aligned}
$$

$$
\lim _{n \rightarrow \infty} A_{n}=\lim _{n \rightarrow \infty} \inf A_{n}=\lim _{n \rightarrow \infty} \sup A_{n},
$$

when $\lim _{n \rightarrow \infty} \inf A_{n}=\lim _{n \rightarrow \infty} \sup A_{n}$.

It is known [1] that these sets are closed.

For a set $A$ in $X$ we denote by $\operatorname{cl} A$ the closure of $A$.

We denote by $R^{m}$ an m-dimensional Euclidean space with the usual norm $|x|$ for each $x \in R^{m}$, and by $I$ the compact interval $\left[t_{0}, t_{0}+a\right]$ in $R^{\mathbf{1}}$.

Let $F(t)$ be a compact and convex set valued measurable function (in $R^{m}$ ) defined on a measurable set $E$.

We denote by $|F|(t)$ a scalar function sup $\{\operatorname{dist}(0, x) ; x \in F(t)\}$. If $|F|(t)$ is integrable on $E$, then the Lebesgue integral $\int_{E} F(t) d t$ has been defined in [2]. In this case we say that $F$ is integrable.

\section{Propositions}

In [3] we have proved the following Propositions. 
Proposition 1. Let $\left\{F_{n}(t)\right\}(n=1,2, \cdots$,$) be a sequence of com-$ pact-set (in $R^{n}$ ) valued functions defined and measurable on $E$ and monotone decreasing in $n$.

Then $\bigcap_{n=1}^{\infty} F_{n}(t)$ is measurable.

Proposition 2. Let $\left\{F_{n}(t)\right\}(n=1,2, \cdots)$ be a sequence of compact-set (in $R^{m}$ ) valued functions defined and measurable on $E$ and $F(t) \subset C(t)(n=1,2, \cdots)$ for some compact-set (in $\left.R^{m}\right)$ valued function $C(t)$. Then $c l \bigcup_{n=1}^{\infty} F_{n}(t)$ is measurable.

Proposition 3. Let $F(t)$ be a compact-set (in $R^{m}$ ) valued function defined on $E$. Suppose that meas $(E)<\infty$.

$F(t)$ is measurable on $E$ if and only if, for every real positive $\varepsilon$, there exists a compact set $E^{\prime}$ in $E$ such that meas $\left(E-E^{\prime}\right)<\varepsilon$ and such that $F(t)$ is upper semi-continuous on $E^{\prime}$.

Remark. In [2] it has been proved that the continuity of $F$ in this sense follows from the measurability of $F$.

Proposition 4. Let $F(t)$ be a compact-set (in $R^{m}$ ) valued function defined and measurable on $E$. Suppose that meas $(E)<\infty$. Then there exists a measurable function $f(t)$ on $E$ such that $f(t)$ $\in F(t)$ for each $t \in E$.

Proposition 5. Let $F(t, x)$ be a compact-set (in $R^{m}$ ) valued function defined on $I \times R^{m}$ and measurable in $t$ for each fixed $x \in R^{m}$ and upper semi-continuous in $x$ for each fixed $t \in I$.

Then $F(t, x(t))$ is measurable in $t$ for each continuous function $x(t) \in R^{m}$.

Remark. Proposition 5 also holds if $I$ is replaced by a compact set.

Further we can prove the following Propositions.

Proposition 6. Let $F(t, x)$ be a compact-set (in $R^{m}$ ) valued function defined on $I \times R^{m}$ and measurable in $t$ for each fixed $x \in R^{m}$ and upper semi-continuous in $x$ for each fixed $t \in I$. 
Then $F(t, x(t))$ is measurable in $t$ for each measurable function $x(t) \in R^{m}$.

Proof. Since $x(t)$ is measurable on $I$, for every real positive $\varepsilon$ we can find a compact set $J$ in $I$ such that $x(t)$ is continuous on $J$. $F(t, x(t))$ is measurable on $J$.

Hence $F(t, x(t))$ is measurable on $I$.

Proposition 7. Let $F(t, u)$ be a compact-set (in $R^{m}$ ) valued function defined on $I \times R^{r}$ and measurable in $t$ for each fixed $u \in R^{r}$ and continuous in $u$ for each fixed $t \in I$.

Then for every compact set $U$ (in $\left.R^{r}\right) F(t, U)$ is a compact-set (in $R^{m}$ ) valued function and measurable in $t$.

Proof. For each fixed $t \in I, F(t, U)$ is a compact set in $R^{m}$. Indeed, let $\left\{x_{n}\right\}$ be a sequence of points in $F(t, U)$. For each $n$ we can select $u_{n} \in U$ such that $x_{n} \in F\left(t, u_{n}\right)$. Since $U$ is a compact set, we can assume that $\left\{u_{n}\right\}$ converges to $u \in U$. From the continuity of $F(t$, $u$ ) in $u$, there is a subsequence of $\left\{x_{n}\right\}$ which converges to some $x \in$ $F(t, u) \subset F(t, U)$.

The measurability of $F(t, U)$ follows from the following relation; $F(t, U)=c l \bigcup_{i=1}^{\infty} F\left(t, u_{i}\right)$, where $\left\{u_{i}\right\}$ is a dense subset of $U$.

Proposition 8. Let $F(t, u)$ be a compact-set (in $R^{m}$ ) valued function defined on $I \times R^{r}$ and measurable in $t$ for each fixed $u \in R^{r}$ and continuous in $u$ for each fixed $t \in I$.

Then $F(t, Q(t))$ is measurable in $t$ for each measurable compact-set valued function $Q(t) \subset R^{r}$.

Proof. We first prove Proposition when $Q(t)$ is continuous. We denote a subdivision of $I$ by $D: t_{0}<t_{1}<\cdots<t_{k}=t_{0}+a$, and $\delta(D)=\max _{0 \leq i \leq k-1}$ $\left(t_{i+1}-t_{i}\right)$. For $t_{i} \leqq t<t_{i+1}$ we define $Q(t ; D)=Q\left(t_{i}\right)$. Let $\left\{D_{n}\right\}(n=1$, $2, \cdots)$ be a sequence of subdivisions of $I$ such that each division point of $D_{n}$ belongs to that of $D_{n+1}$ and $\left\{\delta\left(D_{n}\right)\right\}$ tends to zero as $n \rightarrow \infty$. Also we define $F_{n}(t)=F\left(t, Q\left(t ; D_{n}\right)\right)$, and

$$
I^{\prime}(C)=\bigcup_{N=1}^{\infty} \bigcap_{n=N}^{\infty} I\left(F_{n} \subset \subset C\right\},
$$


where $I\left(F_{n} \subset \subset C\right)$ denotes the set $\left\{t \in I ; F_{n}(t) \subset \subset C\right\} . \quad I\left(F_{n} \subset \subset C\right)$ is measurable for every $n$, and hence $I^{\prime}(C)$ is measurable.

From the continuity of $F(t, x)$ and $Q(t)$, we can show that $I(F$ $\subset \subset C) \subset I^{\prime}(C)$ and $I\left(F \subset \subset C^{\prime}\right) \supset I^{\prime}(C)$ for every compact set $C^{\prime}(\supset$ $\supset C)$. Let $C_{n}(n=1,2, \cdots)$ be the compact set $c l U\left(C, \frac{1}{n}\right)$. From the relations stated above, it follows $I\left(F \subset \subset C_{n+1}\right) \subset I^{\prime}\left(C_{n+1}\right) \subset I(F \subset \subset$ $\left.C_{n}\right)$. Since $I(F \subset C)=\bigcap_{n=1}^{\infty} I\left(F \subset C_{n}\right)=\bigcap_{n=1}^{\infty} I^{\prime}\left(C_{n}\right), F(t, Q(t))$ is measurable on $I$.

When $Q(t)$ is measurable on $I$, for every $\varepsilon>0$ there is a compact set $J$ in $I$ such that meas $(I-J)<\varepsilon$, and $Q(t)$ is continuous on $J$.

Therefore $F(t, Q(t))$ is measurable on $J$ and hence $F(t, Q(t))$ is measurable on $I$.

Propositon 9. Let $Q(x)$ be a compact-set (in $R^{r}$ ) valued function defined on $R^{m}$ and upper semi-continuous in $x \in R^{m}$.

Let $F(x, u)$ be a compact-set (in $R^{m}$ ) valued function defined on $R^{m} \times R^{r}$ and be upper semi-continuous in $(x, u)$.

Then $R(x)=F(x, Q(x))$ is upper semi-continuous in $x$.

Proof. Take any $x_{0} \in R^{m}$, and $\varepsilon>0$. According to the upper semicontinuity of $Q(x), F(x, X) \subset U\left(R\left(x_{0}\right), \varepsilon\right)$ whenever $\operatorname{Dist}\left(X, Q\left(x_{0}\right)\right)<\delta$ and $\left|x-x_{0}\right|<\delta$ for some $\delta(>0)$. We can take $Q\left(x_{0}\right) \cup Q(x)$ as $X$ if $x$ is sufficiently near $x_{0}$. Consequently the relation

$$
R(x)=F(x, Q(x)) \subset F\left(x, Q\left(x_{0}\right) \cup Q(x)\right) \subset U\left(R\left(x_{0}\right), \varepsilon\right)
$$

holds since Dist $\left(Q\left(x_{0}\right) \cup Q(x), Q\left(x_{0}\right)\right)<\delta$ holds for every $x$ sufficiently near $x_{0}$.

Proposition 10. Let $F(t, u)$ be a compact-set (in $R^{m}$ ) valued function defined on $I \times R^{r}$ and measurable in $t$ for each fixed $u \in R^{r}$ and continuous in $u$ for each fixed $t \in I$.

Let $Q(t)$ be a compact-set (in $R^{r}$ ) valued function defined and bounded on $I$ and measurable in $t$. Let $y(t)$ be a measurable function (in $R^{m}$ ) on $I$.

If $\{u ; F(t, u) \ni y(t), u \in Q(t)\}$ is empty nowhere on $I$, then the 
compact-set valued function $F(t)$ (in $R^{r}$ ) defined as

$$
F(t)=\{u ; F(t, u) \ni y(t), u \in Q(t)\}
$$

is compact-set valued function and measurable on $I$.

Proof. From the compactness of $Q(t)$ and upper semi-continuity of $F(t, u)$ in $u, F(t)$ can be verified to be compact for each $t \in I$.

Take a denumerable set of points $\left\{u_{i}\right\}(i=1,2, \cdots)$ which is dense in $R^{r}$, and a monotone decreasing sequence $\left\{\varepsilon_{j}\right\} \downarrow 0(j=1,2, \cdots)$. Denote the following compact set by $F_{i j}(t)$.

$$
\begin{aligned}
& F_{i j}(t)=\left\{u \in\left\{u_{1} \cdots u_{i}\right\} ; \operatorname{dist}(y(t), F(t, u))<\varepsilon_{j}, u \in U\left(Q(t), \varepsilon_{j}\right)\right\} . \\
& F_{i j}(t) \text { is measurable on } I \text {, and the relation }
\end{aligned}
$$

$$
F(t)=\bigcap_{j=1}^{\infty} c l \bigcup_{i=1}^{\infty} F_{i j}(t)
$$

shows that $F(t)$ is measurable on $I$.

Proposition 11. Let $\left\{F_{n}(t)\right\}(n=1,2, \cdots)$ be a sequence of compact-set (in $R^{m}$ ) valued functions defined and measurable on $E$. Suppose that there exists a compact-set (in $R^{m}$ ) valued function $F_{0}(t)$ such that $F_{n}(t) \subset F_{0}(t) \quad(n=1,2, \cdots)$ on $E$. Then $F(t)=\lim _{n \rightarrow \infty}$ sup $F_{n}(t)$ is measurable.

Proof. Since $F_{n}(t) \subset F_{0}(t)$ on $E, \lim _{n \rightarrow \infty} \sup F_{n}(t)$ exists and is a compact set in $R^{m} . \quad F(t)$ is measurable since $F(t)$ can be expressed as follows.

$$
F(t)=\bigcap_{N=1}^{\infty} c l \bigcup_{n=N}^{\infty} F_{n}(t)
$$

on $E$.

Proposition 12. Let $\left\{F_{n}(t)\right\}(n=1,2, \cdots)$ be a sequence of compact and convex set (in $R^{m}$ ) valued functions defined and integrable on $E$, and suppose that there is an integrable function $F_{0}(t)$ (which is a compact and convex set valued function) such that $F_{n}(t) \subset F_{0}(t)$ $(n=1,2, \cdots)$ on $E$, then $\lim _{n \rightarrow \infty} \sup F_{n}(t)$ is integrable and

$$
\lim _{n \rightarrow \infty} \sup \int_{E} F_{n}(t) d t \subset \int_{E} \lim _{n \rightarrow \infty} \sup F_{n}(t) d t \text { holds. }
$$


Proof. By Proposition 2.1 [1] $\lim _{n \rightarrow \infty} \sup F_{n}(t)$ exists for each $t \in E$. Similarly $\lim _{n \rightarrow \infty} \sup \int_{E} F_{n}(t) d t$ exists, since

$$
\int_{E} F_{n}(t) d t \subset \int_{E} F_{0}(t) d t
$$

Let $x$ be any point in $\lim _{n \rightarrow \infty} \sup \int_{E} F_{n}(t) d t$. Then there is a subsequence $\left\{F_{n^{\prime}}(t)\right\}$ such that

$$
\lim _{n \rightarrow \infty} \operatorname{dist}\left(x, \int_{E} F_{n^{\prime}}(t) d t\right)=0
$$

holds. By Proposition 1.3 [1] we can select a further subsequence $\left\{F_{n^{\prime \prime}}(t)\right\}$ such that $\lim _{n \rightarrow \infty} F_{n^{\prime \prime}}(t)=F(t)$ exists for each $t \in E$. By Proposition $3.2[1]$ we conclude that

$$
\lim _{n \rightarrow \infty} \operatorname{Dist}\left(F_{n^{\prime \prime}}(t), F(t)\right)=0 \text {. }
$$

Since

$$
\begin{aligned}
& \operatorname{dist}\left(x, \int_{E} F(t) d t\right) \\
& \leqq \lim _{n \rightarrow \infty} \operatorname{dist}\left(x, \int_{E} F_{n^{\prime \prime}}(t) d t\right)+\lim _{n \rightarrow \infty} \operatorname{Dist}\left(\int_{E} F_{n^{\prime \prime}}(t) d t, \int_{E} F(t) d t\right),
\end{aligned}
$$

and

$$
\lim _{n \rightarrow \infty} \operatorname{Dist}\left(\int_{E} F_{n^{\prime \prime}}(t) d t, \int_{E} F(t) d t\right)=0
$$

$[2]$, then

$$
\operatorname{dist}\left(x, \int_{E} F(t) d t\right)=0
$$

holds.

Hence

$$
x \in \int_{E} \lim _{n \rightarrow \infty} F_{n^{\prime \prime}}(t) d t \subset \int_{E} \lim _{n \rightarrow \infty} \sup F_{n}(t) d t .
$$

Remark. Proposition 12 also holds if the conditions $F_{n}(t) \subset F_{0}(t)$ are replaced by the following conditions; $\left|F_{n}(t)\right| \leqq M(t)$ on $E$ for some integrable scalar function $M(t)$. 


\section{Existence theorem for contingent equation}

Theorem 1. Let the compact and convex set (in $\left.R^{m}\right)$ valued function $F(t, x)$ be defined on a parallelepiped $R\left(\right.$ in $\left.R^{1} \times R^{m}\right): t_{0} \leqq$ $t \leqq t_{0}+a,\left|x-x_{0}\right| \leqq b$, and measurable in $t$ for each fixed $x$, and $u p$ per semi-continuous in $x$ for each fixed $t$. Let there exist a scalar function $M(t)$, integrable on $I=\left[t_{0}, t_{0}+a\right]$, such that

$$
|F(t, x)| \leqq M(t), \int_{t_{0}}^{t_{0}+a} M(t) d t \leqq b
$$

for all $(t, x) \in R$.

Then there is an absolutely continuous function $x(t)$ such that

$$
d x(t) / d t \in F(t, x(t))
$$

for almost all $t$ in $I$, and $x\left(t_{0}\right)=x_{0}$.

Proof. Let $D$ be a subdivision of $I: t_{0}<t_{1}<\cdots<t_{k}=t_{0}+a$. We denote $\max _{0 \leqq i \leq k-1}\left(t_{i+1}-t_{i}\right)$ by $\delta(D)$. Since $F\left(t, x_{0}\right)$ is measurable on $\left[t_{0}, t_{1}\right]$, we can select a measurable function $f_{0}(t)$ such that $f_{0}(t) \in F\left(t, x_{0}\right)$ for each $t \in\left[t_{0}, t_{1}\right]$.

For $t_{0} \leqq t \leqq t_{1}$ we define

$$
x(t ; D)=x_{0}+\int_{t_{0}}^{t} f_{0}(t) d t,
$$

and put

$$
x_{1}=x_{0}+\int_{t_{0}}^{t_{1}} f_{0}(t) d t
$$

Then

$$
\left|x_{1}-x_{0}\right| \leqq \int_{t_{0}}^{t_{1}} M(t) d t \leqq b
$$

holds.

We define inductively $\left\{x_{i}\right\}$ and $\left\{f_{i}(t)\right\}(i=0,1, \cdots n-1)$ as follows. Suppose that we have defined $x_{i}$ such that

$$
\left|x_{i}-x_{0}\right| \leqq \int_{t_{0}}^{t_{i}} M(t) d t
$$

and then for $t_{i} \leqq t \leqq t_{i+1}$ we define

$$
x(t ; D)=x_{i}+\int_{t_{\mathrm{i}}}^{t} f_{i}(t) d t
$$


and put $x_{i+1}=x\left(t_{i+1} ; D\right)$, where $f_{i}(t)$ is a measurable function such that $f_{i}(t) \in F\left(t, x_{i}\right)$ for each $t \in\left[t_{i}, t_{i+1}\right]$. Then

$$
\left|x_{i+1}-x_{0}\right| \leqq \int_{t_{0}}^{t_{i+1}} M(t) d t
$$

holds.

The function $x(t ; D)$ has thus been defined for all $t \in I$. By defining $y(t ; D)=x_{i}$ for $t \in\left[t_{i}, t_{i+1}\right), \quad 0 \leqq i \leqq k-2$, and $y(t ; D)=x_{k-1}$ for $t \in\left[t_{k-1}, t_{k}\right]$

$$
x(t ; D) \in x(\tau ; D)+\int_{\tau}^{t} F(t, y(t ; D)) d t
$$

holds for all $t \in\left[\tau, t_{0}+a\right]$.

Hence $x(t ; D)$ is absolutely continuous, and

$$
\left|x^{\prime}(t ; D)\right| \leqq M(t), x(0 ; D)=x_{0},
$$

hold independently of the choice of $D$.

Let $\left\{D_{n}\right\}(n=1,2, \cdots)$ be a sequence of subdivisions of $I$ such that $\left\{\delta\left(D_{n}\right)\right\}$ tends to zero as $n \rightarrow \infty$. Since $\left\{x\left(t ; D_{n}\right)\right\}$ is equi-continuous on $I$ and satisfies the same initial condition, $\left\{x\left(t ; D_{n}\right)\right\}$ is a normal family. Hence we can select a subsequence of $\left\{x\left(t ; D_{n}\right)\right\}$ (without changing the notation) which converges to a function $x(t)$ uniformly on $I$, and

$$
\left|x^{\prime}(t)\right| \leqq M(t), x(0)=x_{0}
$$

hold.

From the equi-continuity of $\left\{x\left(t ; D_{n}\right)\right\}$ and the construction of $\left\{y\left(t ; D_{n}\right)\right\}$, we conclude that $\left\{y\left(t ; D_{n}\right)\right\}$ also converges to $x(t)$ uniformly on $I$.

From the relation

$$
x\left(t ; D_{n}\right) \in x\left(\tau, D_{n}\right)+\int_{\tau}^{t} F\left(t, y\left(t ; D_{n}\right)\right) d t
$$

and the upper semi-continuity of $F(t, x)$ in $x$ and Proposition 12

$$
\begin{aligned}
x(t) & \in x(\tau)+\lim _{n \rightarrow \infty} \sup \int_{\tau}^{t} F\left(t, y\left(t ; D_{n}\right)\right) d t \\
& \subset x(\tau)+\int_{\tau}^{t} \lim _{n \rightarrow \infty} \sup F\left(t, y\left(t ; D_{n}\right)\right) d t \\
& \subset x(\tau)+\int_{\tau}^{t} F(t, x(t)) d t .
\end{aligned}
$$


Then

$$
d x(t) / d t \in F(t, x(t))
$$

holds for almost all $t$ in $I$ and $x\left(t_{0}\right)=x_{0}$.

Theorem 2. Let $F(t, x)$ be a compact and convex set (in $R^{m}$ ) valued function defined on $I \times R^{m}$, and be measurable in $t$ for each fixed $x \in R^{m}$ and upper semi-continuous in $x$ for each fixed $t \in I$. Suppose that $x \cdot y \leqq C\left(|x|^{2}+1\right)(C>0)$ holds for every $y$ such that $y \in F(t, x)$, where the dot denotes the scalar product, and that $F(t$, $x)$ carries every bounded set in $I \times R^{m}$ into a bounded set in $R^{m}$.

Then for every $x_{0} \in R^{m}$ there exists an absolutely continuous vector function $x(t)$ such that

$$
d x(t) / d t \in F(t, x(t))
$$

for almost all $t$ in $I$, and $x\left(t_{0}\right)=x_{0}$.

Proof. We first prove this theorem under the assumption that $F(t, x)$ is bounded. Similarly as in the proof of Theorem 1 we define $\left\{x\left(t ; D_{n}\right)\right\}$, and $\left\{y\left(t ; D_{n}\right)\right\}$ such that

$$
x\left(t ; D_{n}\right) \in x(\tau, D)+\int_{\tau}^{t} F\left(t, y\left(t ; D_{n}\right)\right) d t .
$$

Since $F(t, x)$ is bounded, $\left\{x\left(t ; D_{n}\right)\right\}$ is a normal family and then $\left\{x\left(t ; D_{n}\right)\right\}$ with $\left\{y\left(t ; D_{n}\right)\right\}$ can be assumed to converge to a function $x(t)$ uniformly on $I$. From Proposition 12 we conclude that

$$
x(t) \in x(\tau)+\int_{\tau}^{t} F(t, x(t)) d t
$$

Hence

$$
d x(t) / d t \in F(t, x(t))
$$

for almost all $t \in I$, and $x\left(t_{0}\right)=x_{0}$.

Next we denote $\left(\left|x_{0}\right|^{2}+1\right) \exp (2 C a)-1$ by $H^{2}(H>0)$.

By taking a sufficiently large $C$, we can assume that $H>1$. Also we define

$$
\bar{F}(t, x)= \begin{cases}F(t, x), & |x| \leqq H \\ F(t, H x /|x|), & |x|>H\end{cases}
$$


$\bar{F}(t, x)$ defined as above can be verified to be measurable in $t$ for each fixed $x \in R^{m}$ and upper semi-continuous in $x$ for each fixed $t \in I$, and is bounded on $I \times R^{m}$. Hence there exists an absolutely continuous function $x(t)$ such that

$$
d x(t) / d t \in \bar{F}(t, x(t))
$$

for almost all $t \in I$ and $x\left(t_{0}\right)=x_{0}$.

For $\bar{F}(t, x)$ the same relation as $F(t, x)$, i.e., $x \cdot y \leqq C\left(|x|^{2}+1\right)$ for every $y \in \bar{F}(t, x)$, holds. From this condition we can conclude that all solutions of

$$
d x / d t \in \bar{F}(t, x) \text { and } x\left(t_{0}\right)=x_{0}
$$

satisfy $|x(t)| \leqq H$ on $I$.

Indeed if $z(t)=|x(t)|^{2}+1$, then $d z(t) / d t \leqq 2 C z(t)$, hence $z(t) \leqq$ $\left(\left|x_{0}\right|^{2}+1\right) \exp (2 C a)$, i.e. $|x(t)| \leqq H$. In $|x| \leqq H, \bar{F}(t, x)$ and $F(t, x)$ coincide. Hence a solution $x(t)$ for

$$
d x / d t \in \bar{F}(t, x), x\left(t_{0}\right)=x_{0}
$$

is also that for

$$
d x / d t \in F(t, x), x\left(t_{0}\right)=x_{0} .
$$

Consequently we have proved the existence of solutions.

Theorem 3. Let $F(t, x)$ satisfy the condition in Theorem 2.

Then for every compact set $K$ in $R^{m}$ the collection of all solutions $x(t)$ of the contingent equation such that $x\left(t_{0}\right) \in K$ is compact in the topology of the uniform convergence.

Proof. Let $\left\{x_{n}(t)\right\}(n=1,2, \cdots)$ be a sequence of solutions. We must show that there exists a subsequence which converges uniformly to a solution. Since

$$
d x_{n}(t) / d t \in F\left(t, x_{n}(t)\right)
$$

for almost all $t \in I$, it follows that

$$
x_{n}(t)-x_{n}(\tau) \in \int_{\tau}^{t} F\left(t, x_{n}(t)\right) d t .
$$

On the other hand $\left\{x_{n}(t)\right\}$ is uniformly bounded and equi-contin- 
uous on $I$. Thus there exists a subsequence (without changing the notation) which converges uniformly to some function $x(t)$, and $x\left(t_{\mathrm{c}}\right)$ $\in K$ holds.

Further, since all the $x_{n}(t)$ satisfy the same Lipschitz condition, their limit $x(t)$ satisfies the same Lipschitz condition.

Hence $x(t)$ is absolutely continuous.

By Proposition 12,

$$
\begin{aligned}
x(t)-x(\tau) & \in \lim _{n \rightarrow \infty} \sup \int_{\tau}^{t} F\left(t, x_{n}(t)\right) d t \\
& \subset \int_{\tau}^{t} \lim _{n \rightarrow \infty} \sup F\left(t, x_{n}(t)\right) d t \\
& \subset \int_{\tau}^{t} F(t, x(t)) d t
\end{aligned}
$$

holds for all $t \in\left[\tau, t_{0}+a\right]$.

Since $x(t)$ is absolutely continuous,

$$
d x(t) / d t \in F(t, x(t))
$$

for almost all $t \in I$. This completes the proof of the theorem.

\section{Existence of optimal control}

In this chapter we shall consider the control problem for the contingent equation and prove the existence of optimal control.

We shall make the following assumptions.

1) $F(t, x, u)$ is a compact-set (in $R^{m}$ ) valued function defined in $I \times$ $R^{m} \times R^{r}$.

2) $F(t, x, u)$ is measurable in $t$ for each fixed $(x, u) \in R^{m} \times R^{r}$, and continuous in $(x, u)$ for each fixed $t \in I$.

3) $F(t, x, u)$ carries every bounded set in $I \times R^{m} \times R^{r}$ into a bounded set in $R^{m}$.

4) $Q(t, x)$ is a compact-set (in $R^{r}$ ) valued function defined in $I \times R^{m}$ and measurable in $t$ for each fixed $x \in R^{m}$, and upper semi-continuous in $x$ for each fixed $t \in I$.

5) $Q(t, x)$ carries every bounded set in $I \times R^{m}$ into a bounded set in $R^{r}$. 
6) $R(t, x)=F(t, x, Q(t, x))=\{y ; y \in F(t, x, u), u \in Q(t, x)\}$ is a compact and convex set (in $R^{m}$ ) for each $(t, x) \in I \times R^{m}$.

7) For every $t$ and $x$ and $u \in Q(t, x), x \cdot y \leqq C\left(|x|^{2}+1\right)$ holds for every $y$ such that $y \in F(t, x, u)$, where the dot denotes the scalar product. 8) $\mathrm{K}$ is a compact set in $R^{m}$. $K(t)$ is a compact-set (in $R^{m}$ ) valued function defined in $I$, and upper semi-continuous in $t$.

9) $f(t, x)$ is a real function defined in $I \times R^{m}$, and is measurable in $t$ for each fixed $x \in R^{m}$, and continuous in $x$ for each fixed $t \in I$, and is bounded from below.

If $u(t)$ is a measurable function in $R^{r}, F(t, x, u(t))$ is measurable in $t$ for each fixed $x \in R^{m}$, and is continuous in $x$ for each fixed $t \in I$. Therefore for each measurable function $u(t)$ the system of equations

$$
\left\{\begin{array}{l}
d x(t) / d t \in F(t, x(t), u(t)) \text { for almost all } t \in I, \\
x\left(t_{0}\right)=x_{0}
\end{array}\right.
$$

has an absolutely continuous solution $x(t)$ for every $x_{0} \in R^{m}$, if $F(t, x$, $u$ ) satisfies the assumptions stated above.

We say that $x(t)$ is the trajectory corresponding to a control $u(t)$ (measurable in $t$ and $\in Q(t, x(t))$ on $I$ ) if $x(t)$ is an $m$-dimensional, absolutely continuous function satisfying the above system of equations.

We say that a control $u(t)$, defined for $t_{0} \leq t \leq \bar{t}, \bar{t} \in I$, transfers $K$ to $K(t)$ if one of the trajectories $x(t)$ corresponding to $u(t)$ satisfies the relations $x\left(t_{0}\right) \in K$ and $x(\bar{t}) \in K(\bar{t})$.

We shall consider the problem of finding a control function $u(t)$ which transfers $K$ to $K(t)$ and which minimizes the cost functional

$$
J(x)=\int_{t_{0}}^{\bar{t}} f(t, x(t)) d t
$$

where $x(t)$ is one of the solutions corresponding to $u(t)$, and $\bar{t}$ represents a value of $t$ such that $x(t) \in K(t)$.

Theorem 4. Suppose that the conditions stated above are satisfied. Also suppose that there exists at least one control $u(t)$ which transfers $K$ to $K(t)$ on $I$.

Then there exists an optimal control, i.e., a measurable func- 
tion $u^{*}(t)$ for which one of the corresponding solutions, $x^{*}(t)$, with initial condition $x^{*}\left(t_{0}\right) K$, attains $K\left(t^{*}\right)$ for some $t^{*}$ in $I$, and

$$
\inf J(x)=J\left(x^{*}\right)=\int_{t_{0}}^{t^{*}} f\left(t, x^{*}(t)\right) d t,
$$

where, in addition, $u^{*}(t) \in Q\left(t, x^{*}(t)\right)$.

Proof. Now consider the set of all the $x(t)$ satisfying

$$
d x(t) / d t \in F(t, x(t), u(t))
$$

almost everywhere on $I, x\left(t_{0}\right) \in K$ and $x(\bar{t}) \in K(\bar{t})$ for some $\bar{t} \in I$, where, in addition, $u(t) \in Q(t, x(t))$ for some control $u(t)$. Since one such solution exists by hypothesis, this set is not empty. Consequently we can select a sequence of trajectories $\left\{x_{n}(t)\right\}$ on $I$, with

$$
J\left(x_{n}\right)=\int_{t_{0}}^{t_{n}} f\left(t, x_{n}(t)\right) d t
$$

decreasing monotonically to inf $J(x)$, where $t_{n}$ represents a value of $t$ such that $x_{n}(t) \in K(t) . \quad x_{n}(t)$ satisfy the following relations

$$
d x_{n}(t) / d t \in R\left(t, x_{n}(t)\right)
$$

almost everywhere on $I$ and $x_{n}\left(t_{0}\right) \in K$. By the compactness of solutions of the contingent equation, we conclude that

$$
d x^{*}(t) / d t \in R\left(t, x^{*}(t)\right), x^{*}\left(t_{0}\right) \in K,
$$

where $x^{*}(t)$ is a limit function of a subsequence of $\left\{x_{n}(t)\right\}$. Also we can select a further subsequence (without changing the notation) such that $\left\{t_{n}\right\}$ converges to some $t^{*}$ in $I$ since $I$ is a compact interval. Further, making use of the equi-continuity of $\left\{x_{n}(t)\right\}$ and the upper semicontinuity of $K(t)$, we conclude that $x^{*}\left(t_{0}\right) \in K$ and $x^{*}\left(t^{*}\right) \in K\left(t^{*}\right)$. From Proposition 8 we can select a measurable function $u^{*}(t)$ such that

$$
d x^{*}(t) / d t \in F\left(t, x^{*}(t), u^{*}(t)\right)
$$

almost everywhere on $I$ and $u^{*}(t) \in Q\left(t, x^{*}(t)\right)$ on $I$. Finally

$$
J\left(x_{n}\right)=\int_{t_{0}}^{t_{n}} f\left(t, x_{n}(t)\right) d t
$$

approaches 
$\int_{t_{0}}^{t *} f\left(t, x^{*}(t)\right) d t$ as $n \rightarrow \infty$ and hence inf $J(x)=J\left(x^{*}\right)$. Thus $x^{*}(t)$ on $t \leq t \leqq t^{*}$ is optimal.

\section{REFERENCES}

[1] M. Hukuhara, Sur 1'application semi-continue dont la valeur est un compact convexe, RIMS-11, Res. Inst. Math. Sci., Kyoto Univ., 1966.

[2] M. Hukuhara, Intégration des applications measurables dont la valeur est un compact convexe, RIMS-15, Res. Inst. Math. Sci., Kyoto Univ., 1966.

[3] N. Kikuchi, Existence of optimal controls, RIMS-20, Res. Inst. Math. Sci., Kyoto Univ., 1967. 
